\title{
Research on robust control of nonlinear singular systems
}

\author{
XuYuting,HuZhen
}

Nanchang Institute of Science \& Technology, Nanchang 330108,China

Keywords: descriptor systems; robust control; scheduling; nonlinear; operations research

\begin{abstract}
For a class of robust problems of uncertain nonlinear singular systems, it has proposed a system robust control method research based on nonlinear programming in the paper, and the uncertainty robust stability analysis model of nonlinear singular systems has been gave. The robust stability of the system is studied by using the nonlinear matrix inequality of operations research. In order to verify the feasibility of the method, a design example for uncertain conditions and planning issues to mobilize the mobile equipment, the use of stochastic programming and robust optimization method of operational research under stochastic demand and path planning of mobile facilities. The test results indicate that this method can make the system have a better robustness and control characteristics, computation time and the number of iterations to reach the satisfactory results, the accuracy of the calculation is also good, which is a new method to study the nonlinear robust control of singular systems.
\end{abstract}

\section{Introduction}

In recent years, singular systems have been widely used in control theory, circuit, economy, machinery and other fields. Because the nonlinear descriptor system with uncertainty can describe the objective system more generally, the stability and robustness of the descriptor system is lower than that of the normal system. The nonlinear programming mathematical programming with nonlinear constraints or objective function, is an important branch of operational research, the operational control study the nonlinear programming theory of generalized system robustness, is a new method for improving the stability and robustness of the system, has important implications for the optimization of complex system.

\section{General situation of Nonlinear Singular Systems}

In order to meet the need of large engineering technology, the paper presents an unconventional mathematical model of system description in the field of modern high technology. Generalized system model was first proposed in power network system, the concept of concern at first, most of the studies focus on nonlinear singular systems, then linear singular systems and existence and uniqueness of the solution are discussed and generalized system was officially started, and is used in large engineering field.

Compared with the traditional system, the meaning of the generalized system is more general. Compared with the traditional system, it has obvious advantages in maintaining the physical characteristics. In general, some semi state variables can be eliminated by changing the system, which can be described by the normal system, but it will lose the sparsity of the matrix of the original system. In many physical and operational research situation, existing algebraic equations, especially the application of some coupling, these occasions using algebraic equations to describe the constraints, so the generalized system is a powerful tool to describe many practical systems, it 
has an important significance to the research. Compared with the traditional system, the different points of the generalized system mainly lie in the following aspects

(1) compared with the uniqueness of the solution of the initial value problem of the normal system, the initial value solution of the singular system has many kinds of situations

For example, there is no solution and infinitely many solutions, in the case of the existence of the solution, there will be a pulse and a jump.

(2) the hierarchy of the generalized system is more obvious, on the one hand, the dynamic characteristics of the system

It is shown that the static characteristics of the system are not static.

(3) there are two extreme points for the generalized system, is a great value, is an infinite extreme point, which has great value for the number of points is $\mathrm{Q}$, the number of infinite extreme point is n-q, infinite extreme point is divided into static and dynamic extremum extremum point for a system, only a very little value $n$.

(4) for normal systems, when the structural parameters of the system are disturbed, the structure is stable, and its robustness is good, but the general system does not have a stable structure, and its robustness is not good.

(5) the normal system satisfies the stability and robustness in the sense of Lypaunov, but the generalized system does not have these characteristics.

According to the characteristics of nonlinear singular systems, the stability and robustness of the system can be controlled by operational research theory in order to obtain a relatively stable nonlinear descriptor system.

\section{Robust optimization model for Nonlinear Singular Systems Based on operational research theory}

The engineering model of the generalized system can be expressed in the form of mathematics, and the differential equation or differential equation is used in the modeling of Generalized Systems

$$
\begin{aligned}
& f(T(\lambda) x(t), x(t), u(t), t)=0 \\
& g(x(t), u(t), y(t), t)=0
\end{aligned}
$$

In the formula (1), $x(t), u(t), y(t)$ are the input and output of the descriptor system and the state of the form, $T(\lambda) x(t)$ is the differential or differential form, $f$ is the matrix function of $T(\lambda) x(t), x(t), u(t), t$, and $g$ is the matrix function of $\mathrm{s} x(t), u(t), y(t), t$, the special form of generalized system is that,

$$
\begin{aligned}
& E(t) \dot{x}(t)=J(x(t), u(t), t) \\
& y(t)=K(x(t), u(t), t)
\end{aligned}
$$

In the formula (2), $J(x(t), u(t), t)$ and $K(x(t), u(t), t)$ is the $\mathrm{N}$-dimensional vector function of $x(t), u(t), t, x(t), u(t), y(t)$ is the state, input and output vectors of appropriate dimensions, $t$ is Time vector. Robust optimization can be established according to the different parameters of nonlinear descriptor systems. The robust control optimization model (ROB) is as follows, 


$$
\begin{aligned}
& {[R O B] \min c^{T} x+\max _{b \in B} Q(x, b)} \\
& \text { s.t. } \quad x \in S
\end{aligned}
$$

In the formula (3), B is the set of uncertain parameters in Nonlinear Singular Systems, $Q(x, b)$ is optimal model for control, $c$ is strategy of operations research,$x$ is operations research linear programming unknowns. In order to verify the effectiveness of the nonlinear generalized system of operational research methods, this model is verified by the operational decision making of mobile facilities. The general control system for mobile devices mentioned in the previous section, the main question is what mobile devices need to be put into use, how to arrange the route and schedule of these mobile devices.

In order to optimize the objective function change, we need to the minimum cost of the customer's demand is really allocated to mobile devices in a static state, this distribution belongs to nonlinear singular systems, in order to make the system has good stability, need to adopt the method of operational research to control the robustness of the system, in order to improve the calculation accuracy of the system.

\section{Optimization Analysis of nonlinear singular systems based on Operational Research}

Nonlinear singular systems in the research field of research mainly involves path optimization and scheduling problems, this time by mobile vehicle equipment as the research object, the order and path of mobile vehicle customer needs to establish scheduling model, the structure of the model as shown in Figure 1.

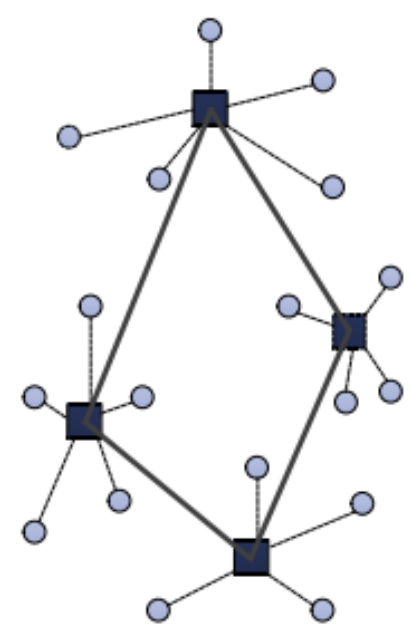

Fig. 1 The mobile device model

The mobile device compared with the traditional equipment, in addition to having similarities, also has the obvious difference, the mobile device is a device that can provide on-demand services, still can provide services for customers around, and the move has obvious uncertainty, is a kind of generalized nonlinear systems. In order to verify the operational research theory in the planning of robust control of singular systems, a generalized model of mobile equipment system of distribution according to need, statistical convergence and calculation of system optimization, the calculation results as shown in Figure 2. 


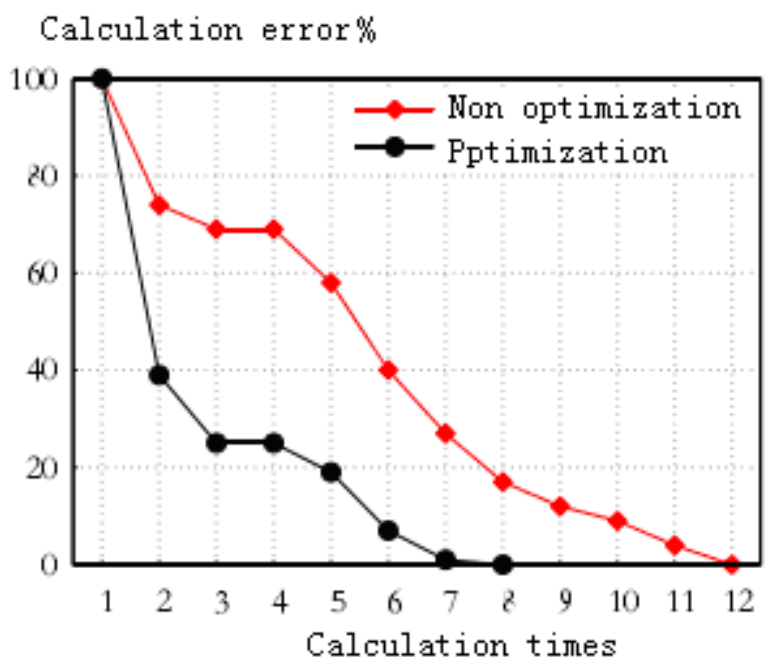

Fig. 2 Robust convergence of the system before and after optimization

Figure 2 shows the research of non-linear programming optimization of robust convergence curves before and after the system theory, the results show that the robust convergence rate of optimization method after the system has been significantly improved, which verifies the validity of the research of nonlinear programming method.

Tab.1 The results of system optimization

\begin{tabular}{|c|c|c|c|}
\hline $\begin{array}{c}\text { Calculation } \\
\text { number }\end{array}$ & $\begin{array}{c}\text { computing } \\
\text { time/s }\end{array}$ & $\begin{array}{c}\text { Iteration } \\
\text { number }\end{array}$ & $\begin{array}{c}\text { Calculation } \\
\text { accuracy / \% }\end{array}$ \\
\hline 1 & 1.21 & 8 & 98.7 \\
\hline 2 & 1.35 & 6 & 97.2 \\
\hline 3 & 1.62 & 7 & 97.6 \\
\hline 4 & 1.51 & 8 & 98.9 \\
\hline 5 & 1.43 & 6 & 98.5 \\
\hline
\end{tabular}

Table 1 shows several system optimization results obtained by using nonlinear programming theory, can be seen from table 1, using the proposed robust optimization of uncertain nonlinear singular systems on mobile devices, the less computation time, iteration times less, and higher calculation accuracy.

\section{Conclusions}

Based on the nonlinear programming theory of operational research, the robustness of a class of uncertain nonlinear singular systems is studied, and the design method of robust controller is given. In order to verify the feasibility of the method, the design example, the test results indicate that this method can make the system has good robust control characteristics, required less time and iteration calculation, provides a new method for the optimization design of nonlinear descriptor systems.

\section{References}

[1] Zhang Xiuyu, Wang Jianguo, Sun Lingfang, et al. Robust adaptive dynamic surface control for a class of strict feedback nonlinear systems with complex nonlinear links. Journal of Northeast Dianli University, 2012,32 (3): 16-20 (in Chinese)

[2] Chen Jia, Fang yangwang, Lou Shuntian. Robust H nonlinear stochastic perturbation system full order observer. Journal of Xi'an Electronic and Science University, 2013,40 (3): 57-65 
[3] Wang Keqi, Yang Zhi. Time delay discrete system with delay dependent Guaranteed Cost Control for uncertain. Information and Control, 2010,39 (003): 373-378

[4] Jin Yajie, Lu Jingchao, Zhou Fei. Helicopter design robust guaranteed cost control law. Computer Measurement \& Control, 2010 (003): 571-574

[5] Li Wei, Wang Yanfei. Fault tolerant guaranteed cost control of NCS robust $\mathrm{H}$ a less conservative. Control and Decision, 2011,26 (12): 1768-1776

[6] Li Wei, Cao Huichao. Uncertain robust $\mathrm{H}$ guaranteed dynamic output feedback fault-tolerant control NCS. Computer Science, 2012,39 (7)

[7] Lun Suxian, Wang Zhongquan. A class of uncertain $\mathrm{H}$ fuzzy control of networked control systems with guaranteed performance control. Journal of Liaoning Technical University (Natural Science), 2010,29 (004): 634-637

[8] Yang Liyun, Research on the direction finding problem based on ant colony algorithm. Computer Engineering and Design, 2009, (10)

[9] Li Guanghua, Liu Aimei, Zhou Guohua. Study on the size of private vehicles in urban distribution center. Railway Transport and Economy, 2007, (12): 61-64

[10] Wang Jing, Zhang Ling, Huang Jun, et al. Robust emergency logistics system based on uncertain demand. Mathematics in Practice and Theory, 2009, (20): 53-60

[11] Zhang Qingling, Wang Qing, Cong Xin. Study on stability of singular systems. Journal of Northeastern University (Natural Science), 2002, 23 (7):624-627

[12] Piao Fengxian, Ma Xiuzhen. Robust stability analysis of uncertain descriptor systems. Journal of Shenyang Aerospace University, 2002, 19:73-75.

[13] Xu Shengyuan, Yang Chengwu. Robust control for a class of uncertain nonlinear singular systems. Control Theory \& Applications, 2000, 17(4):62-625.

[14]Hu Gang, Xie Xiangsheng. Robust stabilization of uncertain descriptor systems based on LMI method. Journal of Guangdong University of Technology, 2001, 18 (2):6-8,30.

[15] Hu Gang, Ren Junchao, Liu Yongqing. Output feedback robust stabilization for uncertain descriptor systems. Journal of South China University of Technology(Natural Science Edition), 2002, 30 (4):48-51. 